\title{
Controlling proteins the intein way
}

Several methods have been described recently that permit researchers to exert posttranslational control over gene products. An intein-based method shows it is a strong contender in the field by demonstrating control of protein activity in a whole animal.

Inteins are curious protein elements that can excise themselves out of a protein and reseal the protein behind them as if they had never been there. Although they are described as parasitic, inteins can function in a split form with each half present on the end of a different protein. The split inteins then ligate such proteins together, creating a new protein with novel functions. This ability has made them an attractive tool for protein engineering.

Tom Muir of Rockefeller University and his colleagues have been keenly focused on exploiting inteins for the purposes of targeted protein engineering and control. They previously described the creation of rapamycin-inducible split inteins for regulated protein ligation (Mootz \& Muir, 2002). Muir says, "We'd initially thought of this as a binary switch, but as we got into it more it became clear to us that it had the potential to
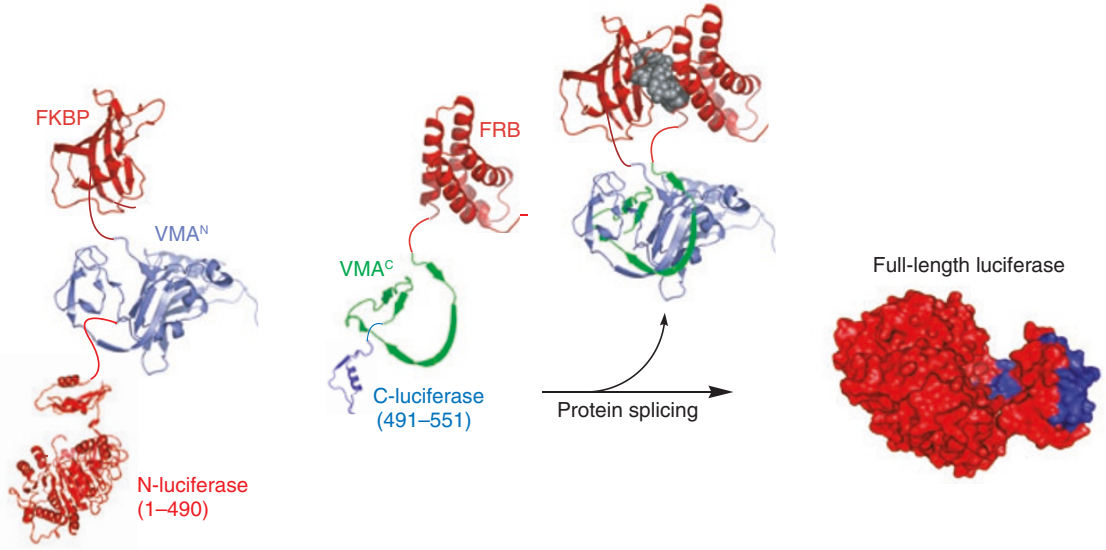

Figure 1 | Inducible protein splicing. The rapamycin-binding proteins FKBP and FRB are fused to split intein domains $\mathrm{VMA}^{\mathrm{N}}$ and $\mathrm{VMA} \mathrm{C}^{\mathrm{C}}$ connected to the $\mathrm{N}$ - and $\mathrm{C}$-terminal pieces of a split luciferase. Addition of rapamycin induces protein splicing, removal of the intein and release of full-length luciferase.

be a rheostat." As most biological processes result from graded changes in protein function over time, they thought that this system could be a useful method for controlling protein function in vivo in a lifelike manner.

As validation of this idea, Muir and his colleagues split the bioluminescent enzyme luciferase into halves and attached their drug-inducible intein domains (Schwartz et al., 2007; Fig. 1). They expressed the proteins in cells and observed that addition of rapamycin resulted in strong dose-dependent activation of luciferase activity. It worked so well they decided to try it in an animal. Muir

\section{SYSTEMS BIOLOGY}

\section{FRETTING FOR A MORE DETAILED INTERACTOME}

A quantitative high-throughput FRET-based method of screening fluorescent protein fusion libraries brings the promise of more detailed interactome maps.

Fluorescence resonance energy transfer (FRET) is a powerful method to quantitatively measure protein-protein interactions in cells, but it has not been applied to high-throughput projects, where it has the potential to provide more detailed quantitative information than traditional methods such as the yeast two-hybrid $(\mathrm{Y} 2 \mathrm{H})$ assay. "Despite the clear utility of library screening, it has been too challenging to try it because of the low efficiency of FRET," explains Patrick Daugherty of the University of California, Santa Barbara.

Using YPet and CyPet fluorescent proteins (Nguyen \& Daugherty, 2005), which had been optimized for intracellular FRET, Daugherty and colleagues now developed a FRET-based assay for screening protein interactions in virtually all types of cells (You et al., 2006). They coexpressed a target protein-YPet fusion and a library of peptide ligands as fusions with CyPet, and then used fluorescenceactivated cell sorting (FACS) to detect cells exhibiting FRET. In bacterial cells, the partly overlapping FRET signals necessitated several rounds of enrichment by FACS, but in yeast and mammalian cells, the large dynamic range in FRET signals between interacting and noninteracting pairs is sufficient for one-step screening of libraries for interaction partners. The researchers were also able to determine the interaction affinities in selected clones by measuring fluorescence intensity as a function of YPet concentration in a lysate dilution series.

One notable result was that the protein-protein interaction affinities measured in vitro for purified proteins were different from those measured in cell lysates. "This is interesting because it begs the question of whether or not in vitro data are sufficient when we develop models and maps for a systems biology perspective. Context-dependent data will probably be required," says Daugherty. He believes that protein crowding is one contributor to this discrepancy and that no existing theory for protein crowding can describe true binding affinity in a crowded environment. "The affinities that we measure in solution using typical methods for purified proteins are just one way to assess binding affinity, but they do not really reflect what is going on inside of the cell." The new FRET-based method is unique because the interaction occurs in the crowded environment of the cell-which has the additional benefit of increasing the specificity of the selection.

Although there are many advantages of using this new method over $\mathrm{Y} 2 \mathrm{H}$ assays, including the ability to detect interactions outside 
says, "It was actually my student Ed Schwartz who bravely took that big leap and moved it into the fly system. We were very happily surprised how straightforward it was." By feeding the fly food laced with rapamycin they could switch luciferase activity on within minutes.

It turns out that the rate-determining step in this technology is getting it to work initially in culture by finding the right points in the target protein to split the molecule. Muir suggests that going forward it may be possible to develop some empirical rules to simplify the process, but there will always be an element of trial and error requiring careful testing and validation. To justify this effort you have to have a problem that can only be addressed by this type of method.

What kinds of applications are these new methods appropriate for? Most likely it will be ones that require titration of protein levels, something that is very difficult to achieve with transcriptional regulation. Muir believes this is going to be the 'killer app' for this kind of technology. "Any system where the protein levels oscillate as a function of some sort of intrinsic or extrinsic control [is] worth exploring with this kind of small molecule-regulated system," says Muir.

"What we'd really like to do with this technology is adapt it such that we get away from small molecules altogether," says Muir. "One thing we are looking at carefully in the lab is trying to control this with light. That would ultimately provide the best level of control." Muir is unwilling to make any bets on which of the recent methods developed to regulate proteins at the post-transcriptional level will ultimately prove the most useful but you can bet his intein-based methods will be contenders.

\section{Daniel Evanko}

\section{RESEARCH PAPERS}

Mootz, H.D. \& Muir, T.W. Protein splicing triggered by a small molecule. J. Am. Chem. Soc. 124, 9044-9045 (2002).

Schwartz, E.C. et al. Post-translational enzyme activation in an animal via optimized conditional protein splicing. Nat. Chem. Biol. 3, 50-54 (2007).

the cell nucleus and in different cell types as well as the ability to quantify binding affinities, it has inherent limitations, being a FRETbased method. The fluorescent proteins must be brought to within about $6 \mathrm{~nm}$ of each other for FRET to occur, and thus the system is limited to studying smaller proteins or parts of proteins.

Daugherty points out that an exciting application of this method would be domain-level protein interaction screening, for example, to build a higher-resolution interaction map after a Y2H screen. The information obtained in a FRET assay-such as which domains interact and their binding affinities-could be used to assemble more detailed protein interaction maps with enough information build models about the system. But to build such a map, a huge effort would be required to examine each of the domains of a given system: first determining which proteins are potentially involved in the system using expression data, then cloning individual domains of the identified proteins and finally screening individual domains against a cDNA fragment library. "That would yield interesting data that are not showing up in the current Y2H screens," adds Daugherty. "We are contemplating whether to jump in or not."

\section{Irene Kaganman}

RESEARCH PAPERS

Nguyen, A.W. \& Daugherty, P.S. Evolutionary optimization of fluorescent proteins for intracellular FRET. Nat. Biotechnol. 23, 355-360 (2005).

You, X. et al. Intracellular protein interaction mapping with FRET hybrids. Proc. Natl. Acad. Sci. USA 103, 18458-18463 (2006).

\section{NEWS IN BRIEF}

\section{IMMUNOCHEMISTRY}

\section{Using plants to make a better antibody}

It is well known that the efficacy of an antibody can be influenced by modifying the glycosylation pattern. Cox et al. describe a new approach to optimize antibody glycan configuration by using a genetically modified aquatic plant, Lemna minor, in which several components of the glycosylation machinery have been silenced. This robust system led to the generation of antibodies with optimized activity, containing only a single major $\mathrm{N}$-glycan species.

Cox, K.M. et al. Nat. Biotechnol. 24, 1591-1597 (2006).

\section{PROTEIN BIOCHEMISTRY}

\section{Experimental validation of computational design}

In contrast to error-prone PCR, a more 'intelligent' way to introduce new function onto protein scaffolds is to use computational tools to design and optimize a library of alternative protein sequences. Few of these libraries, however, have been experimentally characterized. Treynor et al. report the systematic evaluation of seven library-design algorithms with the goal of introducing shifted fluorescence emission to GFP variants. Treynor, T.P. et al. Proc. Natl. Acad. Sci. USA 104, 48-53 (2007).

\section{GENOMICS}

\section{Sorting out Mos1 insertion mutants in worms}

Mutants exist for about a quarter of Caenorhabditis elegans genes. As a complement to other large-scale mutant screens, Duverger et al. present a high-throughput approach to generating Mos1 transposon insertion mutants. Using a combination of fluorescence sorting, a liquid-handling robot and parallel cultivation of several thousand strains in liquid culture, they generated more than 17,500 homozygous insertional mutants.

Duverger, Y. et al. Nucleic Acids Res.; published online 12 December 2006.

\section{IMAGING AND VISUALIZATION}

\section{Imaging bacterial infections in vivo}

Using near-infrared fluorescent zinc(II) dipicolyamine-based dyes that specifically target Gram-positive bacterial cell surfaces, Leevy et al. show that bacterial infections can be imaged in living mice at a tissue depth of more than two centimeters. They introduced the dye into the bloodstream of mice and found that it accumulated at a site of local Staphylococcus aureus infection. Leevy, W.M. et al. J. Am. Chem. Soc. 128, 16476-16477 (2006).

\section{MICROFLUIDICS}

\section{Optimizing experiments the microfluidic way}

Using crystallization trials of membrane proteins as a proof-ofprinciple system, Li et al. demonstrate a simple microfluidics approach for simultaneous screening and optimization of experimental parameters in nanoliter volumes. They combined reagents in a controlled manner in microfluidic channels to generate hundreds of 10-nl droplets, each representing an individual experiment. They obtained protein crystals diffracting at $1.9-2.5 \AA$, validating the approach.

Li, L. et al. Proc. Natl. Acad. Sci. USA 103, 19243-19248 (2006). 